\title{
A Phase III clinical study to evaluate the efficacy of combined azithromycin and dexamethasone in the treatment of blepharoconjunctivitis
}

This article was published in the following Dove Press journal:

Clinical Ophthalmology

9 November 2013

Number of times this article has been viewed

\author{
Kamran Hosseini ${ }^{1,2}$ \\ Judith Hutcheson' \\ Richard L Lindstrom ${ }^{3-5}$ \\ 'Clinical Affairs, ${ }^{2}$ Regulatory Affairs, \\ InSite Vision Incorporated, Alameda, \\ CA, USA; ${ }^{3}$ Minnesota Eye Consultants, \\ Bloomington, MN, USA; ${ }^{4}$ University \\ of Minnesota Department of \\ Ophthalmology, Minneapolis, MN, \\ USA; ${ }^{5}$ University of California, Irvine \\ Gavin Herbert Eye Institute, Irvine, \\ CA, USA
}

Purpose: The purpose of this study was to evaluate the clinical and antimicrobial efficacy and safety of ISV-502 (1.0\% azithromycin and $0.1 \%$ dexamethasone) compared to $1.0 \%$ azithromycin or $0.1 \%$ dexamethasone in the treatment of subjects with blepharoconjunctivitis.

Patients and methods: Patients with verified blepharoconjunctivitis were randomized to receive ISV-502 (Group 1; $\mathrm{n}=140$ ), 1.0\% azithromycin alone (Group 2; $\mathrm{n}=141$ ), or 0.1\% dexamethasone alone (Group 3; $n=136$ ). Bacterial cultures were obtained from the conjunctiva and eyelid. Treatment was instilled in both eyes twice daily at 12-hour intervals for 14 days. The primary endpoint was complete resolution of clinical signs and symptoms at Day 15 . The secondary endpoint was complete bacterial eradication at Day 15 among subjects with positive bacterial cultures at baseline.

Results: Significantly more Group 1 subjects met the primary endpoint (27.1\%) than those in Group 2 (15.6\%; $P=0.028)$, but not compared to Group 3 (23.5\%; $P=0.581)$. Significantly more Group 1 patients $(60 \%)$ had complete bacterial eradication at Day 15 compared with Group 3 (40.2\%; $P=0.007)$, but there was no difference compared with Group 2 (66.3\%; $P=0.306)$. Adverse events were reported in about $25 \%$ of the subjects, with an equal distribution among treatment arms; the most common adverse event was irritation at the instillation site. Visual acuity and intraocular pressure differences were not statistically significant, but did show age and sex differences between groups.

Conclusion: ISV-502 is effective in the treatment of blepharoconjunctivitis as evaluated by clinical cure and bacterial eradication scores. ISV-502 was superior to $1.0 \%$ azithromycin in clinical cure and superior to $0.1 \%$ dexamethasone in bacterial eradication.

Keywords: blepharitis, inflammation, eye

\section{Introduction}

Blepharoconjunctivitis is characterized by inflammation of the outer eyelids and the conjunctiva that results in redness and swelling. It is associated with increased bacterial colonization, especially with Staphylococcus species. It is diagnosed when there is simultaneous occurrence of blepharitis and conjunctivitis, and there are both inflammatory and bacterial components to the disorder. ${ }^{1-3}$ If left untreated, blepharoconjunctivitis may lead to scarring of the eyelid and loss of proper eyelid function over time with secondary damage to the ocular surface. ${ }^{1,2}$ Several subtypes of blepharoconjunctivitis have been identified, including anterior disease, posterior disease (similar to blepharitis), angular blepharoconjunctivitis, seborrheic blepharoconjunctivitis, and atopic blepharoconjunctivitis, ${ }^{1,2}$ and different subtypes may require different treatment strategies. In atopic blepharoconjunctivitis, for
Correspondence: Kamran Hosseini InSite Vision Incorporated,

965 Atlantic Avenue, Alameda, CA 9450I, USA

Tel + I 5108658800

Fax + I 5108655700

Email khosseini@insite.com 
example, an allergic component may be treated successfully with steroids, while a bacterial load would necessitate both antibiotics and steroids for an optimal and quick resolution.

Blepharoconjunctivitis commonly presents as diffuse inflammation of both the anterior and posterior lid margins as well as the conjunctiva and is usually associated with an increase in the local bacterial load. ${ }^{4,5}$ Discharge secondary to blepharoconjunctivitis is not uncommon, and may result in patients reporting eye dryness with eyelids stuck shut in the morning and the presence of greasy and/or crusty eyelashes. Although blepharoconjunctivitis is more common among individuals of middle age or older, it also has been reported in childhood. ${ }^{6}$

In its chronic form, acute onset blepharoconjunctivitis can lead to lid ulceration that results in malposition of tear duct and/or corneal sequelae. Although viral or bacterial microorganisms may cause blepharoconjunctivitis, toxins produced by the Staphylococcal spp. (in particular Staphylococcus epidermis and aureus) have been implicated as a major cause of blepharoconjunctivitis. ${ }^{3,5,7}$ In general, $S$. epidermis and $S$. aureus are found in both the staphylococcal and mixed seborrheic-staphylococcal manifestations. ${ }^{3}$ Other organisms found when culturing the lids and conjunctiva in patients with blepharitis include Propionibacterium acnes and Corynebacteria. ${ }^{7-9}$

A combination agent that addresses both the microbiological and inflammatory components of blepharoconjunctivitis may be highly effective in clearing signs and symptoms while eradicating the inciting organism or group of organisms. ${ }^{8}$ Currently, there is limited efficacy data regarding the available treatments for blepharoconjunctivitis, and there are no approved treatments in the United States. We evaluated the combination of $0.1 \%$ dexamethasone and $1.0 \%$ azithromycin compared to each component alone for the treatment of blepharoconjunctivitis to determine if the combination would provide a greater therapeutic effect (that is, quicker resolution of the disorder) than the individual components used alone. ISV-502 has been studied previously to determine its efficacy against various bacterial loads. ${ }^{10}$ Torkildsen et al ${ }^{11}$ found combining an antibiotic with a steroid to be statistically superior to results with either component alone, although the group evaluated a different antibiotic and had a much smaller sample size. To our knowledge, our study represents the largest controlled clinical study on a potential treatment for blepharoconjunctivitis to date.

\section{Patients and methods}

\section{Study design}

This was a randomized, double-masked, parallel group and comparative Phase III study conducted at 29 different clinical practice sites across the United States. The study was conducted in compliance with all applicable governmental rules, ethical principles, Good Clinical Practice regulations, International Conference on Harmonisation guidelines, and in accordance with the Declaration of Helsinki. The study protocol was reviewed and approved by the Research Consultants Review Committee Institutional Review Board (IRB) (Austin, TX, USA) and Coast IRB (Colorado Springs, CO, USA). It was registered at ClinicalTrials.gov under the registry number NCT01408082.

All enrolled subjects were informed that the study drugs were being used for investigational purposes only. Written informed consent was obtained from all trial participants. Subjects between the ages of 6 and 17 years were required to provide written assent, and a parent or legal guardian was required to provide informed consent for all enrollees younger than 18 years.

\section{Study population}

A total of 417 subjects were assigned to one of three groups: combination $1.0 \%$ azithromycin and $0.1 \%$ dexamethasone (ISV-502; InSite Vision Incorporated, Alameda, CA, USA; Group 1), 1.0\% azithromycin alone (Group 2); or 0.1\% dexamethasone alone (Group 3) in a 1:1:1 ratio according to a randomization schedule generated by the trial's sponsor. At each study site, subjects were randomized sequentially by assigning the number corresponding to the lowest numbered drug kit available at the site. All investigators were masked to the randomization code. All study medications were administered from identical multidose containers so that neither the participant nor the investigator knew which medication the subject was receiving.

Inclusion criteria included a positive diagnosis of blepharoconjunctivitis, defined as a presence of one of the eyelid signs (eg, lid margin redness or swelling), and one of the conjunctival signs (eg, bulbar conjunctival redness, palpebral conjunctival redness, or ocular discharge). In addition, the presence of an ocular symptom was required (eg, lid irritation, itchy eyelids, gritty eyes, or painful/sore eyes). Enrollees had to have a minimum total score of 5 in at least one eye to be eligible for study entry. For subjects who had both eyes qualify for the study, the eye with the highest combined clinical signs and symptoms score on Day 1 of the study 
was analyzed. If the total score was the same in both eyes, the right eye was designated as the study eye.

Other inclusion criteria were best corrected visual acuity (BCVA) $\geq 20 / 100$ in both eyes and an intraocular pressure (IOP) $\leq 25 \mathrm{mmHg}$ in either eye. To participate in the study, subjects had to agree to discontinue systemic antibiotic medication use at least 7 days prior to enrollment through to the end of the study, and subjects also could not have used any corticosteroid medication within 14 days prior to study enrollment. Exceptions were made to allow use of topical antibiotics on the face (except around the eyes) for dermatological conditions; also, the use of inhaled and nasal corticosteroids and topical dermal steroids (except on the eyelids) were permitted. Per study protocol, patients had to discontinue contact lens wear at least 72 hours prior to the first visit and throughout the duration of the study. Patients had to agree to not use eye makeup during the study. Patients had to discontinue use of any nondiagnostic topical ophthalmic solutions, including tear substitutes, and any medication that the investigator deemed may interfere with the study parameters (eg, likely to cause ocular drying).

Exclusion criteria included known sensitivity or poor tolerance to any component of the study medications, history of eyelid and/or ocular surgery in the previous 6 months, or an acute ocular and/or lash or lid infection or active ocular inflammation other than blepharoconjunctivitis. Patients with a history of glaucoma were also excluded from the study. Other exclusion criteria included any uncontrolled systemic disease or debilitating disease (eg, cardiovascular disease, hypertension, diabetes, or cystic fibrosis), any clinically significant cardiovascular disorders (eg, unstable angina, myocardial infarction or cerebrovascular accident within the past 6 months, Class III or IV congestive heart failure, ventricular arrhythmias), any history of liver or kidney disease resulting in persisting dysfunction, or any active upper respiratory tract infection. Women of childbearing potential were required to have a negative pregnancy test prior to their entry into the study. Female subjects who had a hysterectomy, bilateral oophorectomy, bilateral tubal ligation, or who were postmenopausal by at least 12 months or premenarchal were considered as not of childbearing potential.

\section{Treatment}

\section{Planned treatment protocol}

Treatment in the study included combination 1.0\% azithromycin and 0.1\% dexamethasone (ISV-502; Group 1), 1.0\% azithromycin alone (Group 2), or $0.1 \%$ dexamethasone alone (Group 3) instilled in both eyes twice daily at approximately 12-hour intervals for 14 days. Subjects were instructed to place 1 drop of the study medication on his or her eyelid and one drop in the eye at each instillation. The first dose of medication was administered under an investigator's supervision, as was the morning dose during subject visits to the investigator's site. Study participants were responsible for the remaining instillations.

Instructions for administering all other doses at home were provided, and patients were asked to complete a dosing diary. Subjects were instructed to bring the bottles of study medication and subject diary at each planned study visit and return for all subsequent visits between 8 am and $11 \mathrm{am}$.

\section{Identification of study medications}

ISV-502 is a dual-agent investigational study drug comprised of the antibacterial agent $1.0 \%$ azithromycin and the corticosteroid $0.1 \%$ dexamethasone. The antibacterial agent $1.0 \%$ azithromycin is a broad-spectrum antibiotic with activity against gram-positive and gram-negative bacteria. Azithromycin is classified as an azalide, a subclass of macrolide antibiotics, a class of drugs that arrest bacterial cell growth by inhibiting protein synthesis. ${ }^{12,13}$

The anti-inflammatory component of ISV-502, 0.1\% dexamethasone, is a potent corticosteroid commonly prescribed as an ocular anti-inflammatory agent. ${ }^{10}$ It is approved for ocular administration (both topical and periocular), as well as systemically.

A component of ISV-502, AzaSite $^{\circledR}$ (1.0\% azithromycin ophthalmic solution; InSite Vision), was approved for marketing in the US in April 2007 (NDA 50-810). Ophthalmic dexamethasone is currently indicated for steroid responsive inflammatory conditions that include allergic conjunctivitis and selected infective conjunctivitides when the inherent hazard of steroid use is accepted to obtain an advisable diminution in edema and inflammation. ${ }^{14}$ All study medications were formulated with a proprietary, patented polymeric drug delivery system (DuraSite; InSite Vision), which prolongs the drug's residence time on the ocular surface and allows for distribution of the drug into the conjunctiva, cornea, and eyelids for ocular conditions. It also maintains the dexamethasone suspension and increases viscosity upon instillation, allowing for use as both an eye drop and an ointment.

ISV-502 is intended as a long-acting topical eye drop for twice-daily dosing over a 14-day treatment course. 


\section{Per protocol clinical visits}

The per protocol (PP) clinical visits were planned as follows.

\section{Visit I (Day I)}

The investigator performed an initial eye examination, including assessment of BCVA, signs and symptoms of blepharoconjunctivitis in both eyes, biomicroscopy, and undilated ophthalmoscopy in both eyes. A culture of the eyelid and conjunctiva was taken from the study eye. After the culture was obtained, IOP was measured using applanation tonometry. Subjects not meeting the appropriate inclusion criteria were disqualified.

Following the eye examination, ocular culture, and instillation of the first dose of study medication, subjects were instructed to administer the second dose in both eyes at bedtime, and then twice daily (in the morning and evening) for the next 13 days. Subjects were instructed not to dose the medication on the morning of any subsequent planned visit.

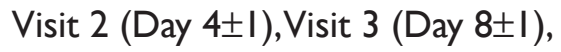
Visit 4 (Day II \pm I)

Ocular symptoms were assessed, followed by BCVA assessment and biomicroscopy in both eyes. A culture of the eyelid and conjunctiva was taken from the study eye; to ensure the dose was taken $\geq 12$ hours prior to culture, subjects were instructed to dose promptly the night before the visit. Following this, IOP was measured in both eyes using applanation tonometry. Subjects were asked to grade the frequency of the signs and symptoms of blepharoconjunctivitis since the previous visit.

When the study visit procedures were completed, the subject had to instill a drop of the study medication in both eyes and one drop on both eyelids at the study site under the guidance of a study staff member. The subject was reminded to continue dosing twice daily through to Day 14 .

\section{Visit 5 (Day I5-16)}

Overall ocular symptoms were assessed first. Final BVCA, biomicroscopy, and undilated ophthalmoscopy examinations were performed in both eyes. A culture of the eyelid and conjunctiva were taken from the study eye, and IOP was measured in both eyes. The subject was asked to grade the frequency of the signs and symptoms of blepharoconjunctivitis since the previous visit. All study medication, used or unused, as well as the subject's compliance diary, were collected.

\section{Outcome measurements}

The primary endpoint of the study was clinical resolution of all clinical signs and symptoms at Visit 5.
The secondary endpoint was bacterial eradication at Visit 5 (defined as the absence of, or no detectable growth of, the bacterial species). Eradication was assessed for the conjunctiva and lid separately and then combined. The earliest study visit when bacterial eradication was achieved was also determined for subjects with complete eradication at Visit 5. The efficacy of achieving the secondary endpoint was analyzed in all patients with a positive bacterial culture at the time of study enrollment (modified intent-to-treat [mITT] analysis) as well as in a population of patients who completed the study PP.

Other efficacy endpoints included clinical resolution of individual signs and symptoms, time at earliest clinical improvement, and reduction in bacterial load. The Investigator's Global Efficacy Rating and Subject's Global Efficacy Rating were used to score the signs and symptoms of blepharoconjunctivitis during Visits 2-5:

- a score of 0 indicated that all signs and/or symptoms of blepharoconjunctivitis were cleared;

- a score of 1 indicated that signs and/or symptoms were still present, but were less severe;

- a score of 2 indicated that signs and/or symptoms were unchanged;

- a score of 3 indicated that the signs and/or symptoms had worsened compared with baseline.

Ocular safety was assessed in all enrolled subjects who received at least one dose of study medication through patient-reported adverse experience, changes in visual acuity, IOP monitoring, and ophthalmic examination. Safety endpoints included adverse events (AEs), discontinuations due to AEs, and changes in visual acuity ( $>2$ line change), IOP (increase of $\geq 10 \mathrm{mmHg}$ from baseline), or ophthalmic examination findings.

Subjects could be withdrawn from the study at any time point if, in the investigator's opinion, the clinical condition had worsened. Treatment compliance was assessed by subject diary in which the subject recorded the date and time of each drug administration, and the investigators reviewed this diary during Visits $2-5$.

\section{Statistical analysis}

Analysis of the proportion of subjects with complete clinical response was performed with Fisher's exact test using normal approximation, or Cochran-Mantel-Haenszel test using investigation site as strata if appropriate. Efficacy was analyzed in all enrolled subjects who received at least one dose of study medication in an intent-to-treat (ITT) analysis, with last observation carried forward (LOCF) where appropriate. Additional efficacy analysis was performed 
in the population of patients who successfully completed the study PP.

The analysis of the proportion of subjects with clinical response for each sign or symptom was performed with Fisher's exact test using normal approximation. Analysis of the Investigator's and Subject's Global Efficacy Ratings were conducted using LOCF data for the ITT population and performed with a chi-square analysis. Clinical improvement of signs and symptoms at Visit 5 (Day 15-16) was assessed using an analysis of covariance (ANCOVA) model adjusting for the sum of ratings at baseline.

The reduction of bacterial load at Visit 5 (Day 15-16) was assessed using an ANCOVA model adjusting for baseline value. If major assumptions were not true, an appropriate transformation of the data was performed, or a nonparametric model was used instead, such as a categorical chi-square analysis.

All analyses described here were conducted using SAS Version 9.1 (SAS Institute, Cary, NC, USA). A $P$ value of 0.05 or less was considered statistically significant for all outcome measures.

\section{Results}

Of the 417 subjects enrolled, Group 1 comprised 140 patients, Group 2 comprised 141 patients, and Group 3 comprised 136 patients. Baseline characteristics are summarized in Table 1. There were no statistical differences noted at baseline between the three groups.

A total of 386 subjects completed the study. Four patients were discontinued due to protocol violations (one each in Group 1 and Group 2, and two in Group 3), and 13 patients were withdrawn because of adverse outcomes. Additionally, one patient was withdrawn at the investigator's discretion (Group 3), and three were lost to follow up (one in Group 2 and two in Group 3).

Positive bacterial cultures were obtained from 301 enrolled eyes (100 Group 1, 104 Group 2, and 97 Group 3) and were analyzed by presence in the cul-de-sac only, on the eyelid only, and for both sites. The most common organisms in the cul-de-sac were methicillin-susceptible Staphylococcus epidermidis (MSSE), methicillin-susceptible S. aureus, and methicillin-resistant S. epidermidis.

Table I Demographics of subjects by treatment group (ITT population)

\begin{tabular}{|c|c|c|c|c|}
\hline Demographic & AzaSite Plus ${ }^{\mathrm{TM}}(\mathrm{N}=\mid 40)$ & AzaSite $^{\circledR}(\mathrm{N}=|4|)$ & Dexamethasone $(\mathrm{N}=136)$ & Total $(\mathrm{N}=4 \mid 7)$ \\
\hline \multicolumn{5}{|l|}{ Age (years) } \\
\hline Mean (SD) & $59.54(19.63)$ & $58.74(20.1 \mathrm{I})$ & $60.63(18.28)$ & $59.63(19.34)$ \\
\hline Median & 61.5 & 61.0 & 62.5 & 62.0 \\
\hline Min-max & $5.0-90.0$ & $7.0-95.0$ & $7.0-88.0$ & $5.0-95.0$ \\
\hline $2-11$ years & $4(2.9 \%)$ & $3(2.1 \%)$ & I (0.7\%) & $8(1.9 \%)$ \\
\hline $12-16$ years & $5(3.6 \%)$ & $4(2.8 \%)$ & $2(1.5 \%)$ & II (2.6\%) \\
\hline $17-64$ years & $70(50.0 \%)$ & $73(51.8 \%)$ & $67(49.3 \%)$ & $210(50.4 \%)$ \\
\hline$\geq 65$ years & $6 \mathrm{I}(43.6 \%)$ & $6 \mathrm{I}(43.3 \%)$ & $66(48.5 \%)$ & $188(45.1 \%)$ \\
\hline Pediatric ( $\leq 16$ years) & $9(6.4 \%)$ & $7(5.0 \%)$ & $3(2.2 \%)$ & $19(4.6 \%)$ \\
\hline \multicolumn{5}{|l|}{ Sex } \\
\hline Male & 49 (35.0\%) & $68(48.2 \%)$ & $58(42.6 \%)$ & 175 (42.0\%) \\
\hline Female & 91 (65.0\%) & $73(5 \mathrm{I} .8 \%)$ & 78 (57.4\%) & $242(58.0 \%)$ \\
\hline \multicolumn{5}{|l|}{ Ethnicity } \\
\hline Hispanic & $21(15.0 \%)$ & $23(16.3 \%)$ & $2 \mathrm{I}(\mathrm{I} 5.4 \%)$ & 65 (I5.6\%) \\
\hline Non-Hispanic & $119(85.0 \%)$ & $118(83.7 \%)$ & $115(84.6 \%)$ & $352(84.4 \%)$ \\
\hline \multicolumn{5}{|l|}{ Race } \\
\hline African American & $8(5.7 \%)$ & 10 (7.1\%) & 12 (8.8\%) & 30 (7.2\%) \\
\hline American Indian & I (0.7\%) & I (0.7\%) & $0(0.0 \%)$ & $2(0.5 \%)$ \\
\hline Asian & $3(2.1 \%)$ & I (0.7\%) & $3(2.2 \%)$ & 7 (1.7\%) \\
\hline Caucasian & $119(85.0 \%)$ & 117 (83.0\%) & $108(79.4 \%)$ & 344 (82.5\%) \\
\hline Other & $9(6.4 \%)$ & $12(8.5 \%)$ & $13(9.6 \%)$ & 34 (8.2\%) \\
\hline \multicolumn{5}{|l|}{ Iris color } \\
\hline Blue & 46 (32.9\%) & 46 (32.6\%) & 47 (34.6\%) & $139(33.3 \%)$ \\
\hline Brown & 61 (43.6\%) & 56 (39.7\%) & 53 (39.0\%) & 170 (40.8\%) \\
\hline Green & $8(5.7 \%)$ & II (7.8\%) & 17 (I2.5\%) & $36(8.6 \%)$ \\
\hline Hazel & $23(16.4 \%)$ & 27 (19.1\%) & 19 (I4.0\%) & 69 (16.5\%) \\
\hline Other & $2(1.4 \%)$ & I (0.7\%) & $0(0.0 \%)$ & $3(0.7 \%)$ \\
\hline
\end{tabular}

Note: AzaSite ${ }^{\circledR}$ and AzaSite Plus ${ }^{\mathrm{TM}}$ manufactured by InSite Vision Incorporated, Alameda, CA, USA.

Abbreviations: ITT, intent-to-treat; max, maximum; Min, minimum; SD, standard deviation. 


\section{Efficacy}

\section{Primary endpoint}

At the last study visit (Visit 5), complete resolution of signs and symptoms was observed in 38 patients in Group 1 (27.1\%), compared with 22 in Group $2(15.6 \%)$, and 32 in Group 3 $(23.5 \%)$ in the ITT analysis with LOCF. A statistically significant greater percentage of patients in Group 1 achieved the primary endpoint compared with Group $2(P=0.0284)$. However, the difference between Group 1 and Group 3 at Visit 5 was not statistically significant $(P=0.5807)$. Table 2 details these differences. Similar results were found in the PP population.

There was no difference in the percentage of patients meeting the primary endpoint when subjects were analyzed according to age (2-11 years, 12-16 years, 17-64 years, and $\geq 65$ years). Significantly more men in Group 1 met the primary endpoint at Visit 4 compared with Group 3 $(P=0.0051)$, but this difference no longer existed by Visit 5. There was a significant difference in the percentage of men meeting the primary endpoint at Visit 5 in Group 1 compared with Group $2(P=0.0153)$; there were no statistically significant differences between group responses in women.

\section{Secondary endpoint}

The secondary endpoint of bacterial eradication is detailed in Table 3. There was a statistically significant greater percentage of patients with bacterial eradication in Group 1 compared with Group 3 starting at Visit 2; this was maintained through Visit 5 (60.0\% versus 40.2\%, respectively; $P=0.0068)$. However, a similar percentage of patients in Group 1 and Group 2 achieved complete bacterial eradication, and there was no statistically significant difference at any time point $(60 \%$ versus $66.3 \%$ at Visit 5, respectively; $P=0.305$ ).
The low number of subjects within the age group stratification (2-11 years, 12-16 years, 17-64 years, and $\geq 65$ years) prevented a complete analysis of the secondary endpoint. However, a statistically significantly greater percentage of patients 17 to 64 years of age in Group 2 met the secondary endpoint compared with Group 1 at Visit 4, but the differences were not statistically significant by Visit 5. In subjects older than 65 years, Group 1 had a higher bacterial eradication than Group 3 at Visits $2-5(P \leq 0.0020)$.

When analyzed by sex, a greater percentage of women in Group 1 compared with Group 3 met the secondary endpoint at Visits $2-5(P \leq 0.0049)$, and among men at Visit 2 only $(P=0.0051)$.

Additional analysis was performed looking at eradication of bacterial culture by eye site. For the cul-de-sac alone, the only significant difference was at Visit 2 between Group 1 and Group 3. For the eyelid alone, there was a statistically significant difference at Visits 2-5 favoring Group 1 versus Group 3 with and without LOCF, similar to findings when both sites were combined.

\section{Other efficacy endpoints}

\section{Signs and symptoms}

Improvement from baseline was observed in all treatment groups, although there were differences in overall resolution in Group 1 compared with Group 2 at Visit $4(P=0.0118)$ and Visit $5(P=0.0077)$. There was no significant difference between Group 1 and Group 3; however, there was a trend favoring Group 1 at Visits 4 and 5.

Overall, there was a difference in resolution of symptoms at Visit 5 among Group 1 patients compared with Group 2 $(P=0.0392)$. Among overall signs, there was a statistically

Table 2 Clinical resolution by treatment group and visit for the ITT population with LOCF

\begin{tabular}{|c|c|c|c|}
\hline Visit/clinical resolution & AzaSite Plus $^{\mathrm{TM}}(\mathrm{N}=140)$ & AzaSite $^{\circledR}(\mathrm{N}=|4|)$ & Dexamethasone $(\mathrm{N}=136)$ \\
\hline \multicolumn{4}{|l|}{ Visit I } \\
\hline Summed score $>0$ & 140 (100\%) & $|4|(100 \%)$ & $136(100 \%)$ \\
\hline \multicolumn{4}{|l|}{ Visit 2} \\
\hline Summed score $>0$ & I 32 (94.3\%) & I 33 (94.3\%) & $123(90.4 \%)$ \\
\hline \multicolumn{4}{|l|}{ Visit 3} \\
\hline Summed score $>0$ & I 34 (95.7\%) & 137 (97.2\%) & 130 (95.6\%) \\
\hline Complete clinical resolution & $5(3.6 \%)$ & $2(1.4 \%)$ & $4(2.9 \%)$ \\
\hline \multicolumn{4}{|l|}{ Visit 4} \\
\hline Summed score $>0$ & II 8 (84.3\%) & $126(89.4 \%)$ & $122(89.7 \%)$ \\
\hline Complete clinical resolution & $2 \mathrm{I}(\mathrm{I} .0 \%)$ & $13(9.2 \%)$ & $12(8.8 \%)$ \\
\hline \multicolumn{4}{|l|}{ Visit 5} \\
\hline Summed score $>0$ & $102(72.9 \%)$ & 117 (83.0\%) & $102(75.0 \%)$ \\
\hline Complete clinical resolution & $38(27.1 \%)$ & $22(15.6 \%)$ & $32(23.5 \%)$ \\
\hline
\end{tabular}

Note: AzaSite ${ }^{\circledR}$ and AzaSite Plus ${ }^{\mathrm{TM}}$ manufactured by InSite Vision Incorporated, Alameda, CA, USA.

Abbreviations: ITT, intent-to-treat; LOCF, last observation carried forward. 
Table 3 Bacterial eradication by treatment group and visit for the mITT population with LOCF

\begin{tabular}{|c|c|c|c|}
\hline Visit/bacterial eradication & AzaSite Plus ${ }^{\mathrm{TM}}(\mathrm{N}=100)$ & $\operatorname{AzaSite}^{\circledR}(\mathrm{N}=104)$ & Dexamethasone $(\mathrm{N}=97)$ \\
\hline \multicolumn{4}{|l|}{ Visit I (Day I) } \\
\hline Bacteria present & $100(100 \%)$ & $104(100 \%)$ & 97 (I00\%) \\
\hline \multicolumn{4}{|l|}{ Bacteria absent } \\
\hline \multicolumn{4}{|l|}{ Visit 2 (Day 4) } \\
\hline Bacteria present & $32(32.0 \%)$ & $27(26.0 \%)$ & $54(55.7 \%)$ \\
\hline Bacteria absent & $64(64.0 \%)$ & 67 (64.4\%) & $31(32.0 \%)$ \\
\hline \multicolumn{4}{|l|}{ Visit 3 (Day 8) } \\
\hline Bacteria present & $27(27.0 \%)$ & $27(26.0 \%)$ & $53(54.6 \%)$ \\
\hline Bacteria absent & 73 (73.0\%) & $73(70.2 \%)$ & $42(43.3 \%)$ \\
\hline \multicolumn{4}{|l|}{ Visit 4 (Day II) } \\
\hline Bacteria present & $35(35.0 \%)$ & $25(24.0 \%)$ & $55(56.7 \%)$ \\
\hline Bacteria absent & $65(65 \%)$ & $76(73.1 \%)$ & $4 \mathrm{I}(42.3 \%)$ \\
\hline \multicolumn{4}{|l|}{ Visit 5 (Day I5) } \\
\hline Bacteria present & $40(40 \%)$ & 33 (31.7\%) & $58(59.8 \%)$ \\
\hline Bacteria absent & $60(60 \%)$ & 69 (66.3\%) & 39 (40.2\%) \\
\hline
\end{tabular}

Notes: Three hundred and one subjects in the ITT population had positive bacterial cultures at baseline and were included in the mITT population. AzaSite ${ }^{\circledR}$ and AzaSite Plus $^{\text {TM }}$ manufactured by InSite Vision Incorporated, Alameda, CA, USA.

Abbreviations: ITT, intent-to-treat; LOCF, last observation carried forward; mITT, modified intent-to-treat.

significant difference between Group 1 and Group 2 at Visit 4 $(P=0.0120)$ and Visit $5(P=0.0132)$.

When analyzed by individual signs and symptoms, there were significant differences among Group 1 patients compared with Group 2 patients in resolution of bulbar conjunctival redness at Visits 4 and 5, itchy eyelids at Visit 5, lid irritation on Visits 3 and 5, and lid swelling at Visits 4 and 5. There were no statistically significant differences in resolution of individual signs and symptoms when Group 1 was compared with Group 3.

\section{Bacterial load}

While bacterial colonization varied among the treatment groups at baseline, there was a significantly greater reduction of bacterial load in Group 1 patients compared with Group 3 patients at Visit $4(P=0.0495)$ and Visit $5(P=0.0239)$. Similarly, the reduction in bacterial load for the mITT population without LOCF showed similar differences in favor of Group 1 compared with Group 3 at Visit $3(P=0.0172)$ and Visit 4 $(P=0.0242)$.

\section{Clinical and microbiological cure}

There was no difference between the percentage of patients with clinical and microbiological cure at Visit 5 with or without LOCF (range, 10.3\%-15.0\%).

\section{Investigator's global rating}

All treatment groups showed improvement or cure over time, and the highest cure rate of $45 \%$ and improvement rate of $35 \%$ was seen in Group 1 at Visit 5, but this was not statistically different compared with other treatment groups.
Similar results were seen in the mITT and PP populations with LOCF.

\section{Subject's global rating}

All treatment groups showed improvement or cure over time, and the highest cure rate of $36.4 \%$ and improvement rate of $52.9 \%$ was seen in Group 1 at Visit 5, but this was not statistically different compared with other treatment groups. Similar results were seen in the mITT and PP populations with LOCF.

\section{Safety}

All study medications were well tolerated, as there was a low incidence of AEs attributable to study medications (Table 4). AEs were reported in about $25 \%$ of participants; most were mild to moderate in severity, and there was an equal distribution among treatment groups. The most frequently reported $\mathrm{AE}$ was instillation site irritation, which was more common in Group 1 and Group 2. Ocular disorders were more common in Group 3. Nasopharyngitis was reported in three subjects in Group 3.

One serious adverse event (SAE) was noted (coronary artery disease) that was not related to the study medication. A total of 13 subjects discontinued for AEs, including the patient with the SAE. Three subjects in Group 1 and two in Group 3 may have withdrawn for reasons likely attributable to study medications, including allergic reaction, burning upon instillation, increased IOP, and irritation.

Visual acuity was unchanged or improved at Visit 5 compared with Visit 1 in $91.4 \%, 87.9 \%$, and $89.0 \%$ in Group 1, Group 2, and Group 3, respectively (Table 5). Only one 
Table 4 Most frequent adverse events by treatment group ( $\geq 1 \%)$

\begin{tabular}{|c|c|c|c|}
\hline Adverse event & $\begin{array}{l}\text { AzaSite } \\
\text { Plus }^{\mathrm{TM}} \\
(\mathbf{N}=\mid \mathbf{4 0})\end{array}$ & $\begin{array}{l}\text { AzaSite }^{\circledR} \\
(\mathrm{N}=\mid 4 I)\end{array}$ & $\begin{array}{l}\text { Dexamethasone } \\
(\mathrm{N}=136)\end{array}$ \\
\hline \multicolumn{4}{|l|}{ Eye disorders } \\
\hline Eye disorder & $3(2.1 \%)$ & 0 & $6(4.4 \%)$ \\
\hline Visual acuity reduced & $2(\mathrm{I} .4 \%)$ & $2(1.4 \%)$ & $2(1.5 \%)$ \\
\hline Punctate keratitis & $3(2.1 \%)$ & 0 & $2(1.5 \%)$ \\
\hline Vision blurred & $2(1.4 \%)$ & 0 & $2(1.5 \%)$ \\
\hline Conjunctiva edema & 0 & $2(1.4 \%)$ & 0 \\
\hline Eye discharge & 0 & $2(1.4 \%)$ & 0 \\
\hline Eyelid edema & $2(1.4 \%)$ & 0 & 0 \\
\hline \multicolumn{4}{|l|}{ Gastrointestinal disorders } \\
\hline Nausea & $2(1.4 \%)$ & I (0.7\%) & I (0.7\%) \\
\hline \multicolumn{4}{|l|}{ GD\&A } \\
\hline Instillation site irritation & $13(9.3 \%)$ & $17(12.1 \%)$ & $3(2.2 \%)$ \\
\hline Instillation site reaction & $5(3.6 \%)$ & $4(2.8 \%)$ & $5(3.7 \%)$ \\
\hline Instillation site pain & $5(3.6 \%)$ & $2(1.4 \%)$ & I (0.7\%) \\
\hline Instillation site pruritus & $3(2.1 \%)$ & $2(1.4 \%)$ & I (0.7\%) \\
\hline \multicolumn{4}{|l|}{ Infections and infestations } \\
\hline Nasopharyngitis & 0 & 0 & $3(2.2 \%)$ \\
\hline Influenza & 0 & $2(1.4 \%)$ & 0 \\
\hline Sinusitis & 0 & $2(1.4 \%)$ & 0 \\
\hline \multicolumn{4}{|l|}{ Injury, poisoning, procedure } \\
\hline Foreign body in eye & 0 & 0 & $2(1.5 \%)$ \\
\hline \multicolumn{4}{|l|}{ Nervous system disorder } \\
\hline Headache & $2(1.4 \%)$ & $2(1.4 \%)$ & 0 \\
\hline
\end{tabular}

Note: $\mathrm{AzaSite}^{\circledast}$ and AzaSite Plus ${ }^{\mathrm{TM}}$ manufactured by InSite Vision Incorporated, Alameda, CA, USA.

Abbreviation: GD\&A, general disorders and administration site.

participant each in Group 1 and Group 3 had worsening of three lines or more at Visit 5 . The majority of patients had no change on ophthalmoscopy or on slit lamp biomicroscopy examination.

At all follow-up visits, the mean change in IOP was higher in Group 1 and Group 3 than in Group 2. Median

Table 5 Summary of change in visual acuity from baseline for worst eye (safety population)

\begin{tabular}{|c|c|c|c|}
\hline $\begin{array}{l}\text { Visit/change in } \\
\text { visual acuity }\end{array}$ & AzaSite Plus $^{\mathrm{TM}}$ & AzaSite $^{\circledR}$ & Dexamethasone \\
\hline \multicolumn{4}{|l|}{ Visit 5 - Visit I } \\
\hline-3 or less & $0(0.0 \%)$ & I (0.7\%) & $0(0.0 \%)$ \\
\hline-2 & $3(2.1 \%)$ & I (0.7\%) & I (0.7\%) \\
\hline-1 & $18(12.9 \%)$ & $22(15.6 \%)$ & $19(14.0 \%)$ \\
\hline 0 & $76(54.3 \%)$ & $73(51.8 \%)$ & $72(52.9 \%)$ \\
\hline I & $28(20.0 \%)$ & $24(17.0 \%)$ & 27 (19.9\%) \\
\hline 2 & $3(2.1 \%)$ & $3(2.1 \%)$ & $2(1.5 \%)$ \\
\hline 3 or more & I (0.7\%) & $0(0.0 \%)$ & I (0.7\%) \\
\hline $\begin{array}{l}\text { No change or } \\
\text { better (line } \\
\text { change }<3 \text { ) }\end{array}$ & 128 (9|.4\%) & 124 (87.9\%) & $|2|(89.0 \%)$ \\
\hline
\end{tabular}

Note: AzaSite $^{\circledR}$ and AzaSite Plus ${ }^{\mathrm{TM}}$ manufactured by InSite Vision Incorporated, Alameda, CA, USA.
IOP change was zero in Group 2 and $1.0 \mathrm{mmHg}$ in the other two groups. Among Group 1 patients, the change in mean IOP compared with baseline was $0.89 \pm 2.11 \mathrm{mmHg}$ at Visit 2 and $1.25 \pm 2.78 \mathrm{mmHg}$ at Visit 5. In Group 3, the mean IOP change increased $1.18 \pm 2.34 \mathrm{mmHg}$ at Visit 2 and $1.04 \pm 2.66 \mathrm{mmHg}$ at Visit 5. Only one patient experienced an IOP spike of more than $10 \mathrm{mmHg}$, but the patient was discontinued after an unrelated incident of herpes zoster ophthalmia.

\section{Treatment compliance}

Treatment compliance was assessed by the subject's recording the date and time of each drug instillation; this data was reviewed by study staff at all follow-up visits. The subject was also questioned at each visit to determine if the subject had followed the dosing schedule. The first instillation of medication and the morning instillation of the medication at Visits 2-4 were administered under supervision in the clinic and recorded. Noncompliance with study protocol based on diary data was a reason for study discontinuation.

The subjects were instructed to bring all study medications (used and unused) and subject diaries to each visit and to return medication at Visit 5 (Day 15-16). On Visit 5 (Day 15-16), all subject medication and the subject diaries were returned when the subjects exited the study.

\section{Discussion}

This randomized, double-masked comparative study found ISV-502 (combination arm) to be effective in the treatment of blepharoconjunctivitis and to be superior to each of its individual active ingredients in the ability to achieve both clinical resolution of the signs and symptoms (compared to azithromycin 1\%), and bacterial eradication (compared to dexamethasone $0.1 \%$ ) at Visit 5 . Although there were differences among the treatment groups in the incidence of positive bacterial cultures, this was likely due to a small number of subjects with pathogens isolated, as the highest incidence was $17.3 \%$ for MSSE in Group 2 compared with $11.0 \%$ in Group 1 and 13.4\% in Group 3. These same organisms and resistant types were also predominant in the eyelid only, but the incidences were greater; the highest incidence was for MSSE in Group 3 (43.3\%) compared with $42.0 \%$ in Group 1 and $35.6 \%$ in Group 2.

Of note, bacterial eradication was sufficiently achieved at Day 8 (Visit 3) within the arms containing an antibiotic (combination arm, and azithromycin arm), and the eradication results did not significantly improve by Day 15, which was the end of the dosing period. It would be interesting to 
gauge the clinical improvement at the same visit and examine whether there is any correlation between bacterial eradication and clinical improvement. However, this study was designed only to evaluate the complete resolution of signs and symptoms (total score of 0 ) at each visit. As an example, those patients that had achieved $90 \%$ clinical resolution were not counted as a "success."

While visual acuity was evaluated primarily for safety in the worse eye only (Table 5), there was an improvement of one to three lines of visual acuity in approximately $15 \%$ of subjects across all groups, regardless of the administered investigational drug. One could speculate that if this analysis had been done in the better eye, that a larger percentage per group would demonstrate improved visual acuity from baseline to Visit 5.

Anecdotal evidence suggests a hard course of combination therapy for 7 days followed by a long-term maintenance therapy, may be efficacious in treating blepharoconjunctivitis. Our study analyzed only full remission data, and we therefore cannot compare our outcomes to those with a shorter course of therapy. Generally speaking, eye care professionals aim to reduce both the bacterial and inflammatory aspects of the disorder, which limits the efficacy of either an antibiotic or a corticosteroid if used alone. ${ }^{15}$ Other studies have evaluated azithromycin $1 \%$ in the treatment of blepharoconjunctivitis, and outcomes have been mixed. ${ }^{16-19}$ The published literature also includes studies with higher dosing frequency than has been suggested here. ${ }^{11}$

As noted, the combination arm in this study proved superior to the other two arms in both bacterial eradication and clinical resolution of signs and symptoms of blepharoconjunctivitis. From a clinical perspective, this study can be considered a success; from a regulatory perspective, that is not the case. The US Food and Drug Administration (FDA) advocates the combination of improved clinical signs and symptoms as its primary endpoint for the treatment of blepharoconjunctivitis. Bacterial eradication is considered a secondary endpoint only. As our study results found the primary endpoint met in both the investigational product but also in the corticosteroid arm, this limits the regulatory definition of "trial success." There is some weight to the US FDA's perspective, as a small number of patients presenting with blepharoconjunctivitis diagnosis proved to be culture negative. In real-world practice, however, the majority of clinicians prefer to address both bacterial load and clinical signs and symptoms to provide patients the broadest and most efficacious treatment.
This study has some limitations. Bacterial load was variable at baseline, potentially impacting interpretation of treatment effect on bacterial colonization. Because blepharoconjunctivitis can recur with some frequency, we recommend a longer-term study that would evaluate the recurrence rates to determine if ISV-502 is an effective prophylactic treatment as well.

In conclusion, ISV-502 was effective in the treatment of blepharoconjunctivitis as evaluated by clinical cure, with reduction in associated signs and symptoms and significant bacterial eradication at Visit 5 compared with baseline. This study supports twice-daily dosing with a combination of $1 \%$ azithromycin and $0.1 \%$ dexamethasone for $8-15$ days in the treatment of blepharoconjunctivitis.

\section{Acknowledgments}

This paper was presented in part at the Association for Research in Vision and Ophthalmology annual conference, 2011. Michelle Dalton provided medical writing and editing expertise.

\section{Author contributions}

$\mathrm{KH}$ and JH conducted the study and analyzed the data; RLL was the clinical advisor of the study; all authors prepared, reviewed, and approved the manuscript.

\section{Disclosure}

This study was sponsored by InSite Vision Incorporated, which designed and conducted the original studies. $\mathrm{KH}$ and JH are employees of InSite Vision. RLL is a consultant for InSite Vision, Alcon, and Bausch + Lomb, and is a member of the scientific advisory board of InSite Vision.

\section{References}

1. McCulley JP, Dougherty JM. Bacterial aspects of chronic blepharitis. Trans Ophthalmol Soc U K. 1986;105 (Pt 3):314-318.

2. McCulley JP, Dougherty JM, Deneau DG. Classification of chronic blepharitis. Ophthalmology. 1982;89(10):1173-1180.

3. McCulley JP. Blepharoconjunctivitis. Int Ophthalmol Clin. 1984;24(2): 65-77.

4. Rubenstein JB, Virasch V. Conjunctivitis: Infectious and noninfectious. In: Yanoff M, Duker JS, editors. Ophthalmology. 3rd ed. London: Elsevier, 2009:227-236.

5. Durand ML. Periocular infections. In: Mandell GL, Bennett JE, Dolin R, editors. Mandell, Douglas, and Bennett's Principles and Practice of Infectious Diseases. 7th ed. London: Elsevier, 2010:1569-1575.

6. Hammersmith KM, Cohen EJ, Blake TD, Laibson PR, Rapuano CJ. Blepharokeratoconjunctivitis in children. Arch Ophthalmol. 2005;123(12):1667-1670.

7. Groden LR, Murphy B, Rodnite J, Genvert GI. Lid flora in blepharitis. Cornea. 1991;10(1):50-53.

8. Jackson WB. Blepharitis: current strategies for diagnosis and management. Can J Ophthalmol. 2008;43(2):170-179. 
9. Tetz MR, Klein U, Völcker HE. [Staphylococcus-associated blepharokeratoconjunctivitis. Clinical findings, pathogenesis and therapy]. Ophthalmologe. 1997;94(3):186-190. German [with English abstract].

10. Si EC, Hutcheson J, Hosseini K. Antimicrobial efficacy of azithromycin/ dexamethasone combination in blepharoconjunctivitis patients, a Phase 3 study. Invest Ophthalmol Vis Sci. 2010;51:E-Abstract 2888.

11. Torkildsen GL, Cockrum P, Meier E, Hammonds WM, Silverstein B, Silverstein S. Evaluation of clinical efficacy and safety of tobramycin/ dexamethasone ophthalmic suspension $0.3 \% / 0.05 \%$ compared to azithromycin ophthalmic solution $1 \%$ in the treatment of moderate to severe acute blepharitis/blepharoconjunctivitis. Curr Med Res Opin. 2011;27(1):171-178.

12. Karch AM. Lippincott's Nursing Drug Guide. Philadelphia: Lippincott Williams and Wilkins, 2005.

13. Ianaro A, Ialenti A, Maffia $\mathrm{P}$, et al. Anti-inflammatory activity of macrolide antibiotics. J Pharmacol Exp Ther. 2000;292(1):156-163.
14. Maxidex (dexamethazone) [package insert]. Fort Worth, TX: Alcon Laboratories, Inc, 2003.

15. Lemp MA, Nichols KK. Blepharitis in the United States 2009: a surveybased perspective on prevalence and treatment. Ocul Surf. 2009; 7(Suppl 2):S1-S14.

16. Haque RM, Torkildsen GL, Brubaker K, et al. Multicenter open-label study evaluating the efficacy of azithromycin ophthalmic solution $1 \%$ on the signs and symptoms of subjects with blepharitis. Cornea. 2010;29(8):871-877.

17. John T, Shah AA. Use of azithromycin ophthalmic solution in the treatment of chronic mixed anterior blepharitis. Ann Ophthalmol (Skokie). 2008;40(2):68-74.

18. Luchs J. Efficacy of topical azithromycin ophthalmic solution $1 \%$ in the treatment of posterior blepharitis. Adv Ther. 2008;25(9): $858-870$.

19. Luchs J. Azithromycin in DuraSite for the treatment of blepharitis. Clin Ophthalmol. 2010;4:681-688.
Clinical Ophthalmology

\section{Publish your work in this journal}

Clinical Ophthalmology is an international, peer-reviewed journal covering all subspecialties within ophthalmology. Key topics include: Optometry; Visual science; Pharmacology and drug therapy in eye diseases; Basic Sciences; Primary and Secondary eye care; Patient Safety and Quality of Care Improvements. This journal is indexed on

\section{Dovepress}

PubMed Central and CAS, and is the official journal of The Society of Clinical Ophthalmology (SCO). The manuscript management system is completely online and includes a very quick and fair peer-review system, which is all easy to use. Visit http://www.dovepress.com/ testimonials.php to read real quotes from published authors. 